\title{
Validation of the Brazilian version of the Clinical Gait and Balance Scale and comparison with the Berg Balance Scale
}

\author{
Validação da versão brasileira da escala de \\ marcha e equilíbrio e comparação com a escala de equilíbrio de Berg \\ Jussara Almeida Oliveira Baggio', Mônica de Biagi Curtarelli', Guilherme Riccioppo Rodrigues', Vitor Tumas
}

\begin{abstract}
Objective: To validate the Clinical Gait and Balance Scale (GABS) for a Brazilian population of patients with Parkinson's disease (PD) and to compare it to the Berg Balance Scale (BBS). Methods: One hundred and seven PD patients were evaluated by shortened UPDRS motor scale (sUPDRSm), Hoehn and Yahr (HY), Schwab and England scale (SE), Falls Efficacy Scale International (FES-I), Freezing of Gait Questionnaire (FOG-Q), BBS and GABS. Results: The internal consistency of the GABS was 0.94 , the intra-rater and inter-rater reliability were 0.94 and 0.98 respectively. The area under the receiver operating characteristic (ROC) curve was 0.72 , with a sensitivity of 0.75 and specificity of 0.6 , to discriminate patients with a history of falls in the last twelve months, for a cut-off score of 13 points. Conclusions: Our study shows that the Brazilian version of the GABS is a reliable and valid instrument to assess gait and balance in PD.
\end{abstract}

Key words: Parkinson's disease, balance, gait, validation studies.

RESUMO

Objetivo: Validar a Escala Clínica de Marcha e Equilíbrio (GABS) para a população brasileira com doença de Parkinson (DP) e compará-la com a Escala de Equilíbrio de Berg (BBS). Métodos: 107 pacientes com diagnóstico de DP foram avaliados através das escalas versão reduzida da UPDRS motora, Hoehn e Yahr, Schwab e England, Escala de Medo de Quedas Internacional, Escala de Congelamento da Marcha, GABS e BBS. Resultados: A consistência interna da escala foi 0,94, a confiabilidade inter-examinador 0,98 e intra-examinador 0,94. Curva ROC (receiver operating characteristic curve) de 0,72, com sensibilidade de 0,75 e especificidade de 0,60 para discriminar pacientes com história de queda nos últimos 12 meses, para uma nota de corte de 13 pontos. Conclusão: Nosso estudo demonstra que a versão brasileira da GABS é válida para avaliar a marcha e o equilíbrio em pacientes com DP.

Palavras-Chave: doença de Parkinson, equilíbrio, marcha, estudos de validação.

Parkinson's disease (PD) is a chronic progressive neurological disorder characterized by resting tremor, muscular rigidity, and bradykinesia. In more advanced stages, further axial motor symptoms become evident, such as stooped posture and balance and gait abnormalities, including festination and freezing of gait $(\mathrm{FOG})^{1,2}$.

In addition to the worsening of motor symptoms, patients usually develop cognitive decline and other non-motor problems which can impair the ability to deal with multiple simultaneous tasks and reveal the insufficiency of compensatory motor mechanisms ${ }^{3,4}$.
As a consequence, patients with PD are at an increased risk of falls compared to healthy elderly people ${ }^{5}$. In the long term, most of the survivors experience falls and around $1 / 3$ sustain fractures ${ }^{6}$. Gait and balance difficulties significantly affect the quality of life of patients with PD, and frequent falls are associated with greater disease severity and increased caregiver burden b $^{5,7}$.

In this scenario, the analysis of balance and gait in patients with PD is an essential step in the context of the clinical evaluation. The Berg Balance Scale (BBS) is one of the most commonly used clinical scales for this purpose and has been

\footnotetext{
'Department of Neuroscience and Behavior, University of São Paulo, Ribeirão Preto Medical School, Ribeirão Preto SP, Brazil.

Correspondence: Jussara Almeida Oliveira Baggio; Department of Neuroscience and Behavior, School of Medicine at Ribeirão Preto, Universidade de São Paulo; 14049-900 Ribeirão Preto SP - Brasil; E-mail: jussaraoliv@usp.br

Support: CAPES (Coordenação de Aperfeiçoamento de Pessoal de Nível Superior)

Conflict of interest: There is no conflict of interest to declare.

Received 16 September 2012; Received in final form 22 April 2013; Accepted 29 April 2013.
} 
validated to many languages, including Portuguese ${ }^{9,10}$. The BBS was found to discriminate 'fallers' from 'non-fallers' ${ }^{\text {'11, }}$, although the scale was not specifically designed to evaluate patients with PD and does not screen for gait disturbances.

Recently, the Clinical Gait and Balance Scale (GABS) was created and validated to specifically evaluate patients with PD. The scale is divided into two parts; the first consisting of information about the patient's history and the second measuring gait and balance parameters including relevant items of balance, posture, FOG, gait cycle, and timed gait tasks $^{13}$, therefore, this new instrument may be a better clinical assessment tool for balance and gait in PD patients than BBS. Accordingly, the aim of the present study is to validate a Brazilian version of the GABS and to compare it to the BBS considering the evaluation of patients with PD.

\section{METHODS}

\section{Cross-Cultural Adaptation \\ of the Clinical Gait and Balance Scale}

After authorization given by the original authors, the GABS was translated by two native Portuguese speakers (J.A.O.B. and V.T.) well acquainted with the English language. The two translated versions were compared and adapted so that a consensual Portuguese version was obtained. The resulting version was then back-translated into English by a native speaker familiarized with the Portuguese language and who had had no previous contact with the original English version of the scale. The back-translated version was then compared to the original version of the GABS by the translators, who examined the differences and made consensual modifications in the Portuguese final version. Additional scale adaptations were not necessary during the phase of data collection because all participants did not have difficult to understand the questions.

\section{Patients and controls}

We evaluated a convenience sample of 107 patients with a diagnosis of PD according to the UK Brain Bank diagnostic criteria $^{14}$ that consecutively attended the Movement Disorders Outpatient Clinic of the Ribeirão Preto Medical School. Patients were excluded from the sample due to the following reasons: inability to walk independently, other motor disorders than PD (e.g., resulting from stroke), dementia according to DSM-IV criteria, severe concomitant systemic illness, acute disorder or injury, severe sensorial deficits (e.g., blindness) or evident peripheral neuropathy or orthopedic conditions that could interfere with gait or balance, and other balance disorders unrelated to PD.

We also evaluated 80 age-matched healthy volunteers without history of neurological disorders, acute injury, balance problems, sensorial deficits or orthopedic conditions that could interfere with gait or balance, and non-demented according to the DSM-IV criteria. This control group was composed of persons accompanying patients who attended the Outpatient Clinic of the Ribeirão Preto Medical School and by subjects of communitarian groups of elderly people from the city of Ribeirão Preto.

This study was approved by the University of São Paulo's ethics committee (Comitê de Ética em Pesquisa Protocol number 2.913/2008). Written informed consent was obtained from all of the subjects.

\section{Clinical Assessment}

The patients were all evaluated during the ON phase, firstly by a neurologist that rated the patient's signs and symptoms using a shortened version of the Unified Parkinson's Disease Rating Scale (sUPDRS), the Hoehn and Yahr staging $(\mathrm{HY})^{15}$, and the Schwab and England functional scale (SE) ${ }^{16}$. The sUPDRS included a motor score that measured the same motor signs as the Short Parkinson's Evaluation Scale, but with the original five-point score of the UPDRS ${ }^{17}$. This shortened version of the UPDRS motor scale (sUPDRSm) was shown to have good reliability and validity in Brazilian patients with $\mathrm{PD}^{18}$.

Next, one of the authors (J.A.O.B.) interviewed the patients and recorded demographic and clinical information, including the history of falls and near falls over the previous twelve months.

Near falls was defined as an event on which an individual felt they are going to fall but did not actually fall and a fall was defined as an event which results in a person coming to rest unintentionally on the ground or other level ${ }^{15}$.

The same examiner administered the Freezing of Gait Questionnaire (FOGQ) ${ }^{19}$ and the Falls Efficacy Scale International (FES-I) ${ }^{20,21}$. The FOGQ was designed to assess the severity of freezing in PD patients. The FES-I has already been validated for the Brazilian population and is tool that measures the concern about falling during the performance of a range of daily-life activities ${ }^{21}$. At last, patients were evaluated by the same examiner with the Brazilian versions of the BBS and the GABS. To assess the reliability of the Brazilian version of the GABS, 10 patients were also evaluated on the same day by another examiner for inter-rater reliability analysis, and another 10 patients were evaluated again by the same examiner one week later to assess the intra-rater reliability.

Healthy volunteers were evaluated with the same protocol.

\section{Statistical Analysis}

Descriptive statistics was used to analyze the clinical and demographic characteristics of all participants. To compare the results of the PD and control groups, the Mann-Whitney test was used and the level of statistical significance was set at $\mathrm{p}<0.05$.

The internal reliability of the Brazilian version of the GABS was evaluated by calculating Cronbach's alpha for the whole scale and for its subscales. Values $>0.70$ 
were considered acceptable. Test-retest reliability was assessed using intra-class correlation coefficients (ICC) and values $\geq 0.70$ were considered acceptable for inter-and intra-rater reliability.

Convergent validity was evaluated by means of Spearman's rank correlation coefficient $\left(\mathrm{r}_{\mathrm{s}}\right)$. Additional analyses were performed involving the area under the receiver operating characteristic) ROC curve, sensitivity, specificity, and positive and negative predictive values comparing those patients that have fallen during the last 12 months with those who have not.

The GABS and the BBS were also evaluated regarding their effectiveness in differentiating patients with PD as the disease progresses. The patients were classified according to the HY scale and the Kruskal-Wallis test was used to determine whether there were differences between the stages of the disease. Groups in different stages of PD were compared two-by-two with the Mann-Whitney test in order to check which groups differed, and the same test was used to compare the subgroup of PD patients with mild symptoms (HY stages 1 and 1.5) and healthy controls.

Factor analyses were also performed for the GABS and BBS. Initially, sampling adequacy was measured with the Kaiser-Meyer-Olkin (KMO) test, with values close to 1 considered as indicating greater suitability of the data for factor analysis, and Bartlett's test of sphericity (BTS) was used to test the hypothesis of correlation between the variables. Factor analysis itself was performed using the method of principal components with Varimax rotation of the type and number of factors extracted following the criteria of Kaiser, in which the number of extracted factors is equal to the number of eigenvalues $\geq 1$.

\section{RESULTS}

The clinical and demographic characteristics of the patients and healthy controls are presented in Table 1. The sample of 107 PD patients (62\% males) had a median age of 62 years (range 33-83), median of 7 years of disease duration (range 3-28), median HY stage of 2 (range 1-4), and median SE score of 80 (range 30-100). The sample of 80 controls (52\% males) had a median age of 61.9 years (range 30-81). Falls over the previous 12 months were reported by $43 \%$ of PD patients and $23 \%$ of controls.

The internal consistency of the total score in the Brazilian version of the GABS was 0.94. Item analyses indicated that the exclusion of any of the items of the scale would not reduce alpha to below 0.94 . The 'history' subscale and the performance items had internal consistency values of 0.83 and 0.93, respectively. Intra-rater ICC was 0.94 and inter-rater ICC was 0.98 .

The correlation coefficients between the GABS and the other clinical evaluations are summarized in Table 2. GABS score was moderately or strongly correlated with the scores of the BBS $\left(r_{s}=-0.93 ; p<0.001\right)$, the Pull test $\left(r_{s}=0.60 ; p<0.001\right)$, the HY $\left(r_{s}=0.69 ; p<0.001\right)$, the SE $\left(r_{s}=-0.60 ; p<0.001\right)$, and the FES-I ( $\left.r_{s}=0.61 ; p<0.001\right)$.

The area under (AUC) the ROC curve was calculated to evaluate the reliability of the scale to discriminate patients with a history of falls in the last twelve months. The AUC for the total GABS score was 0.72 (Figure), with a sensitivity of 0.75 , a specificity of 0.6 , a positive predictive value of 0.80 , and a negative predictive value of 0.56 for a cut-off score of 13 points. The AUC for the total score of the BBS was 0.67 (Figure), with a sensitivity of 0.68 , a specificity of 0.63 ,

Table 1. Clinical and demographic characteristics of Parkinson's disease patients and healthy controls.

\begin{tabular}{|c|c|c|c|c|c|}
\hline & \multicolumn{2}{|c|}{ PD patients } & \multicolumn{2}{|c|}{ Healthy controls } & \multirow{2}{*}{$\mathrm{p}$-value } \\
\hline & Mean (SD) & Median & Mean (SD) & Median & \\
\hline Age & $62.1(11.7)$ & 62 & $61.9(10.08)$ & 62 & 0.66 \\
\hline Disease duration & $8.03(4.9)$ & 7 & - & - & - \\
\hline Hoehn and Yahr stage & $2.21(0.79)$ & 2 & - & - & - \\
\hline Schwab and England scale & 79.9 (13.3) & 80 & - & - & - \\
\hline sUPDRSm & $12.5(6.5)$ & 11 & $0.49(1.05)$ & 0 & $0.00001 *$ \\
\hline Number of falls in 12 months & $3.06(8.5)$ & 0 & $0.4(0.8)$ & 0 & $0.005^{\star}$ \\
\hline FOGQ & $5.68(5.08)$ & 4 & $0.01(0.11)$ & 0 & $0.00001 *$ \\
\hline FES-I score & $26.3(13.2)$ & 19 & $18.7(4.5)$ & 18 & $0.002^{*}$ \\
\hline BBS score & $48.6(8.2)$ & 52 & $55.3(1.7)$ & 56 & $0.00001 *$ \\
\hline GABS & $19.45(15.08)$ & 16 & $2.55(3.55)$ & 0 & $0.00001 *$ \\
\hline \multicolumn{6}{|l|}{ GABS timed tasks } \\
\hline Walking 5 meters & $8(4.21)$ & 6.64 & $5.2(1.06)$ & 5.11 & $0.00001^{\star}$ \\
\hline Number of steps & $12.54(4.98)$ & 11 & $9.55(1.21)$ & 10 & $0.00001 *$ \\
\hline Cadence & $1.63(0.25)$ & 1.67 & $1.86(0.25)$ & 1.86 & $0.00001 *$ \\
\hline Walking as fast as possible ( 5 meters) & $6.03(3.24)$ & 5.27 & $3.94(0.78)$ & 3.81 & $0.00001 *$ \\
\hline Stand-walk-sit (10 meters) & $21.26(10.03)$ & 18.13 & $13.64(3.13)$ & 13.03 & $0.00001 *$ \\
\hline
\end{tabular}

*Mann-Whitnney test: significant level<0.05; sUPDRSm: shortened Unified Parkinson's Disease Rating Scale motor; FOG-Q: freezing of gait questionnaire; FES-I: falls efficacy scale international; BBS: Berg balance scale; GABS: Gait and Balance scale; SD: standard deviation. 
a positive predictive value of 0.43 , and a negative predictive value of 0.25 for a cut-off score of 51 .

GABS and BBS scores were able to discriminate patients in the early phase of the disease (HY stages 1 and 1.5) from healthy controls. We found that the scores of patients in the two scales were significantly different from those of healthy controls $(\mathrm{p}=0.01)$.

The total score in the GABS was able to differentiate patients in HY stages 1 to 2 from patients in stages 2.5 to $4(p=0.001)$, whereas the BBS was only able to differentiate patients in stage 4 from the others $(p=0.02)$.

The factor analysis of the GABS yielded a KMO value of 0.81 and the hypothesis of no correlation between variables was rejected with BTS, with $\mathrm{p}=0.0001$. According to Kaiser's criteria, seven factors were found that explained $70.25 \%$ of the total data variance. The examination of the seven factors showed that one main factor comprised items related to balance and gait and explained most of the variance of the scale

Table 2. Correlation coefficients for the gait and balance scale

\begin{tabular}{|c|c|c|}
\hline & Correlation Coefficients $\left(r_{s}\right)$ & $p$-value \\
\hline Age & 0.35 & $<0.001 *$ \\
\hline Disease's duration & 0.30 & $0.001^{*}$ \\
\hline BBS & -0.93 & $<0.001 *$ \\
\hline sUPDRSm & 0.49 & $<0.001 *$ \\
\hline Pull test & 0.60 & $<0.001 *$ \\
\hline HY stage & 0.69 & $<0.001 *$ \\
\hline SE score & -0.60 & $<0.001 *$ \\
\hline FOGQ & 0.37 & $<0.001 *$ \\
\hline FES-I & 0,61 & $<0.001 *$ \\
\hline Number of falls & 0.37 & $<0.001^{*}$ \\
\hline Near falls & -0.24 & $0.011^{*}$ \\
\hline
\end{tabular}

*GABS score significantly correlated; BBS: Berg balance scale; sUPDRSm: shortened Unified Parkinson's Disease Rating Scale motor; HY: Hoehn and Yahr; SE: Schwab and England Scale; FOG-Q: Freezing of gait questionnaire; FES-I: Falls efficacy scale international.

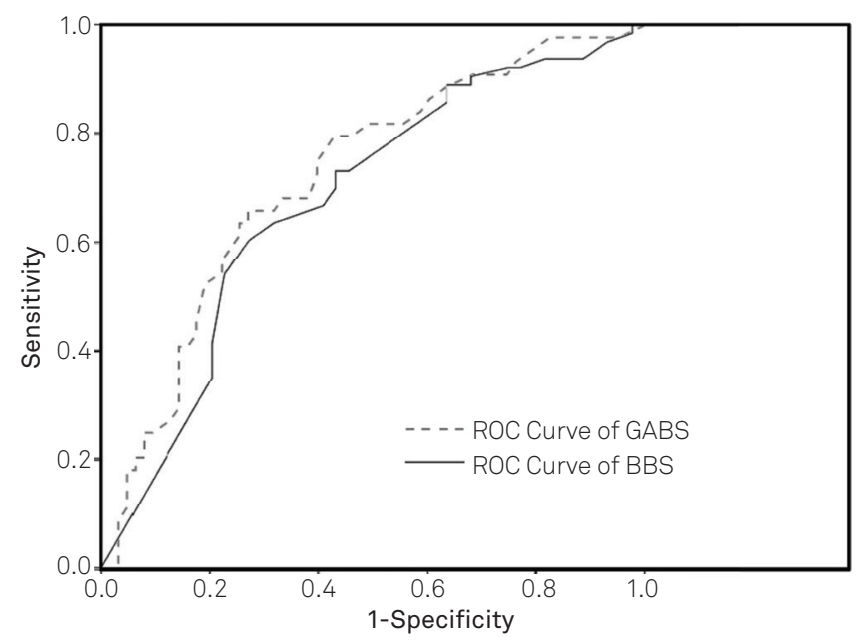

GABS: gait and balance scale; BBS: berg balance scale.

Figure. Receiver operating characteristics curves between patients with and without history of falls in the last twelve months.
(30\%). Two factors encompassed items assessing FOG, while the remaining factors were mainly items also related to balance and gait.

For the BBS, the factor analysis yielded a KMO value of 0.88 and the hypothesis of no correlation between variables was also rejected through BTS, with $\mathrm{p}=0.0001$. Three factors were found according to Kaiser's criteria, which explained $77.45 \%$ of the total variance. However, after analyzing the correlation values between the items, we found that the scale would be better defined by one single factor related to balance.

\section{DISCUSSION}

The GABS was specifically developed to evaluate gait and balance in patients with PD. Our study showed that it provides a reliable quantitative assessment of these clinical parameters in PD patients.

The Brazilian version of the GABS had satisfactory internal consistency, good test-retest reliability, and good sensitivity and specificity to identify patients at risk of falls. Additionally, GABS scores had good correlations with measures of balance, disease severity, and functional independence. These results are consonant with the view that balance and gait disturbances are strongly associated with disability and poor quality of life in patients with mild to moderate $\mathrm{PD}^{8}$.

Balance deficits and falls are frequent clinical problems in patients with $\mathrm{PD}^{5,22}$. In our study, $43 \%$ of $\mathrm{PD}$ patients reported falls in the last 12 months, with previous studies reporting prevalence rates between 19 and 73\%,3,24. This large variability can be explained by differences among the studies. The studies that related more frequency of falls, the patients were older and had greater disease's severity compared with our study. Others differences were the non-exclusion of patients with dementia or the exclusion of falls related to freezing.

We found that the GABS score was poorly correlated with the total number of previous falls. However, the scale was able to discriminate patients who related falls in the last twelve months from patients who did not related falls. This suggests that the scale is more reliable to detect the presence or absence of history of falls, but not to measure the relative risk of falls.

The onset of balance deficits in PD patients is a controversial issue. Frenklach et al. ${ }^{25}$ did not find differences in static and dynamic posturography between controls and subjects in very early stages of PD. In contrast, McVey et al. ${ }^{26}$ observed significant differences in balance recovery variables, used to quantify the response to a backwards waist pull, between PD patients without clinically diagnosed postural instability and healthy controls. In our study, the GABS and BBS were able to discriminate patients with mild symptoms from the control group, demonstrating that both scales were able to detect minimal balance and gait changes in the early stages of PD. 
Our findings showed that the GABS is more effective than the BBS in differentiating patients at different stages of the disease according to the HY classification. The GABS was unable to differentiate patients at each stage of the classification, but was reliable to discriminate those already presenting postural instability. In our study, however, the BBS failed to demonstrate the same power.

The use of the BBS to evaluate balance in PD and other populations is questioned by some authors ${ }^{27,28}$. The analysis of BBS item characteristics for patients with PD suggest revising some BBS item scoring and/or including new items of appropriate difficulty to measure more accurately balance challenges in this population. This better understanding of the BBS at the item level and improvements in its scaling properties would be particularly relevant when BBS scores are used to measure balance progress in the rehabilitation of PD patients ${ }^{27,28}$.

Some of the findings of our study also suggest that the GABS could be more useful than the BBS to evaluate patients with PD. The main advantage of the GABS would consist of increased accuracy to discriminate patients with previous falls and those at different stages of the disease. One of the reasons for this is that the GABS evaluates gait and balance, and not solely balance as the BBS. The factor analysis showed that the GABS evaluates freezing as one factor and gait and balance as another main factor. The BBS has only one factor that evaluates balance. The failure to identify a clear distinction between items evaluating gait and balance in the factor analysis of the GABS could be seen as a drawback of the scale if the aim is to evaluate only one of these factors. However, it can be argued that the factor analysis may not have been able to discriminate between the balance-and gait-related items of the scale, since they can be strongly correlated as shown in our study. Therefore, the appropriateness of the GABS depends on the objectives of the clinical evaluation, while it seems to be quite useful to evaluate several aspects of gait and balance together in patients with PD. The GABS can also evaluate clinical aspects associated with the risk of falls in PD patients and it is a relevant tool to be used in clinical trials and prospective studies ${ }^{11,12}$.

The original study by Thomas et al. ${ }^{13}$ validated the GABS using two computerized and objective measures of balance and gait, that is, the Balance Master and the GAITRite. Our study complements these results by demonstrating that the scale reliably measures gait, balance, and FOG as evaluated by clinical tools, and that the GABS is a valid instrument to evaluate balance and gait in patients with PD. Other studies are needed to evaluate the sensitivity of the scale to detect changes after specific treatments or as a function of the progression of the disease.

One limitation of our study is the absence of a comprehensive cognitive evaluation in both PD and control groups, since cognitive abnormalities may impact balance and gait. Although relevant cognitive changes in PD patients usually occur in older patients ${ }^{29,30}$ (the mean age of our sample was 62 year-old), it is possible that our data have increased the discriminative power of GABS. Hence, the impact of cognitive abnormalities on GABS scores must be addressed in additional studies.

Although we had a control group, we only controlled the variables age and gender. The absence of a general evaluation may compromise the comparison of this group with the PD group. The years of study is an important variable, but we believe that it not influence the final results because all information was collected by the examiners and none patient had difficult to answers the questions.

The absence of a sample calculation did not influence the final results because the statical power of our sample, calculated by Cohen's $d$, was excellent.

In conclusion, our study suggests that the Brazilian version of the GABS is a properly designed, easy-to-use clinical scale and a reliable and valid instrument to assess gait and balance in patients with PD.

\section{References}

1. Jankovic J. Parkinson's disease: clinical features and diagnosis. J Neurol Neurosurg Psychiatry 2008;79:368-376.

2. Bloem BR, Hausdorff JM, Visser JE, et al. Falls and freezing of gait in Parkinson's disease: a review of two interconnected, episodic phenomena. Mov Disord 2004;19:871-884.

3. Fuller RL, Van Winkle EP, Anderson KE, et al. Dual task performance in Parkinson disease: A sensitive predictor of impairment and disability. Parkinsonism Relat Disord 2013;19:325-328.

4. Boonstra TA, van der KH, Munneke M, et al. Gait disorders and balance disturbances in Parkinson's disease: clinical update and pathophysiology. Curr Opin Neurol 2008;21:461-471.

5. Ashburn A, Stack E, Pickering RM, et al. A community-dwelling sample of people with Parkinson's disease: characteristics of fallers and non-fallers. Age Ageing 2001;30:47-52.

6. Hely MA, Reid WG, Adena MA, et al. The Sydney multicenter study of Parkinson's disease: the inevitability of dementia at 20 years. Mov Disord 2008;23:837-844.
Schrag A, Hovris A, Morley D, et al. Caregiver-burden in parkinson's disease is closely associated with psychiatric symptoms, falls, and disability. Parkinsonism Relat Disord 2006;12:35-41.

8. Soh SE, McGinley JL, Watts JJ, et al. Determinants of health-related quality of life in people with Parkinson's disease: a path analysis. Qual Life Res 2012; Epub ahead of print.

9. Miyamoto ST, Lombardi Junior I, Berg KO, et al. Brazilian version of the Berg balance scale. Braz J Med Biol Res 2004;37:1411-1421.

10. Qutubuddin AA, Pegg PO, Cifu DX, et al. Validating the Berg Balance Scale for patients with Parkinson's disease: a key to rehabilitation evaluation. Arch Phys Med Rehabil 2005;86:789-792.

11. Landers MR, Backlund A, Davenport J, et al. Postural instability in idiopathic Parkinson's disease: discriminating fallers from non-fallers based on standardized clinical measures.J Neurol Phys Ther 2008;32:56-61.

12. Dibble LE, Lange M. Predicting falls in individuals with Parkinson disease: a reconsideration of clinical balance measures. J Neurol Phys Ther 2006;30:60-67. 
13. Thomas M, Jankovic J, Suteerawattananon M, et al. Clinical gait and balance scale (GABS): validation and utilization. J Neurol Sci 2004;217:89-99.

14. Hughes AJ, Daniel SE, Kilford L, et al. Accuracy of clinical diagnosis of idiopathic Parkinson's disease: a clinico-pathological study of 100 cases.J Neurol Neurosurg Psychiatry 1992;55:181-184.

15. Hoehn MM, Yahr MD. Parkinsonism: onset, progression and mortality. Neurology 1967;17:427-442.

16. Schwab R, England A. Projection technique for evaluating surgery in Parkinson's disease. In Third Symposium on Parkinson's disease. Toronto, Canadá 1969:152-157.

17. Marinus J, Visser M, Stiggelbout AM, et al. A short scale for the assessment of motor impairments and disabilities in Parkinson's disease: the SPES/SCOPA. J Neurol Neurosurg Psychiatry 2004;75:388-395.

18. Tumas V, Ujikawa LT, Ferreira GM. Utility and reliability of a simplified clinical scale for Parkinson's disease. Arq Neuropsiquiatr 2004;62:S220-S221.

19. Giladi N, Shabtai H, Simon ES, et al. Construction of freezing of gait questionnaire for patients with Parkinsonism. Parkinsonism Relat Disord 2000;6:165-170.

20. Yardley L, Beyer N, Hauer K, et al. Development and initial validation of the Falls Efficacy Scale-International (FES-I). Age Ageing 2005;34:614-619.

21. Camargos FF, Dias RC, Dias JM, et al. Cross-cultural adaptation and evaluation of the psychometric properties of the Falls Efficacy
Scale-International Among Elderly Brazilians (FES-I-BRAZIL). Rev Bras Fisioter 2010;14:237-243.

22. Kim SD, Allen NE, Canning CG, et al. Postural instability in patients with Parkinson's disease : epidemiology, pathophysiology and management. CNS Drugs 2013;27:97-112.

23. Pickering RM, Grimbergen YA, Rigney $U$, et al. A meta-analysis of six prospective studies of falling in Parkinson's disease. Mov Disord 2007;22:1892-1900.

24. Hiorth YH, Lode K, Larsen JP. Frequencies of falls and associated features at different stages of Parkinson's disease. Eur J Neurol 2013;20:160-166.

25. Frenklach A, Louie S, Koop MM, et al. Excessive postural sway and the risk of falls at different stages of Parkinson's disease. Mov Disord 2009; 24:377-385.

26. McVey MA, Stylianou AP, Luchies CW, et al. Early biomechanical markers of postural instability in Parkinson's disease. Gait Posture 2009;30:538-542.

27. Kornetti DL, Fritz SL, Chiu YP, et al. Rating scale analysis of the Berg Balance Scale. Arch Phys Med Rehabil 2004;85:1128-1135.

28. La Porta F, Caselli S, Susassi S, et al. Is the berg balance scale an internally valid and reliable measure of balance across different etiologies in neuroreabilitation? A revisited rash analysis study. Arch Phys Med Rehabil 2012;93:1209-1216.

29. Dubois B, Pillon B, Sternic N, et al. Age-induced cognitive disturbances in Parkinson's disease. Neurology 1990;40:38-41.

30. Muslimovic D, Post B, Speelman JD, et al. Cognitive profile of patients with newly diagnosed Parkinson disease. Neurology 2005;65:1239-1245. 